\title{
Study of the Soret effect in hydrocarbon chain/aromatic compound mixtures
}

\author{
Pavel Polyakov, ${ }^{1, *}$ Eddie Rossinsky ${ }^{2, \dagger}$ and Simone Wiegand ${ }^{1, \ddagger}$ \\ ${ }^{1}$ Forschungszentrum Jülich GmbH, \\ IFF - Weiche Materie, D-52428 Jülich, Germany \\ ${ }^{2}$ Eduard-Zintl-Institut für Anorganische und Physikalische Chemie, \\ Technische Universität Darmstadt, \\ Petersenstrasse 20 D-64287 Darmstadt, Germany
}

(Dated: July 26, 2009)

\begin{abstract}
We studied the thermal diffusion behavior of equimolar mixtures of hydrocarbon chains in cyclohexane, $p$-xylene and $o$-xylene experimentally and by reverse nonequilibrium molecular dynamics. The hydrocarbon chains heptane, 2,3-dimethylpentane, 2,4-dimethylpentane, 2-ethyl 3 methyl butadiene and 2,4 dimethylpentadiene - 1,3 with the same number of carbon atoms were considered in order to conserve the mass contribution and to investigate the shape influence on the Soret coefficient. Compared to the experimental data, the simulation results show the same trend. With increasing degree of branching the Soret coefficient becomes larger. The negative Soret coefficient of the hydrocarbon chains indicates the enrichment in the warm side. We were able to find a empirical correlation between the properties of the pure components and the Soret coeffcients of the mixture.
\end{abstract}




\section{INTRODUCTION}

In a binary mixture exposed to a temperature gradient, the Soret effect induces a concentration gradient.

$$
\nabla x=-S_{\mathrm{T}} x(1-x) \nabla T
$$

$S_{\mathrm{T}}=D_{\mathrm{T}} / D$ is the ratio of the thermal diffusion coefficient and the collective diffusion coefficient. A positive Soret coefficient of the component with the mole fraction $x$ implies that this component moves to the cold region of the fluid.

Although the discovery of the effect by Ludwig took place more than 150 years ago, there is so far only a limited microscopic understanding for liquids ${ }^{1}$. Apparently, the magnitude as well as the sign of $S_{\mathrm{T}}$ are very sensitive to the chosen mixture. Generally, there is no Soret effect in the mixture of absolutely equal components due to the principle of symmetry. The Soret effect is basically the response of the system to the difference between two mixing partners. This simple conception was investigated in detail by experiments and by simulations.

Molecular dynamics simulations of equimolar mixtures of particles ${ }^{2}$ and spherical molecules $^{3}$ show that the component with the larger mass, the smaller radius and the larger depth of the interaction potential moves to the cold side. In our previous work ${ }^{4}$ we have shown experimentally and by reverse non-equilibrium molecular dynamic simulation (RNEMD) that the binary mixtures of simple molecules (tetraethylsilane, di-tert-butylsilane and carbon tetrabromide in carbon tetrachloride) obey this rule of thumb.

Another large group of substance studied are the hydrocarbons which have been investigated experimentally and theoretically ${ }^{5-11}$. However, for alkane/benzene mixtures ${ }^{8}$ the simple rule of thumb fails. The heavier linear alkane always moves to the warm side. This tendency becomes weaker with increasing degree of branching and the highly branched isomer of heptane (2,2,3 - trimethybutane). The trend that linear alkanes have the strongest tendency to move to the warm side had already be observed by Kramers and Broeder for $n$ nonane and $n$-hexadecane ${ }^{5}$ compared to other hydrocarbons, such as the branched isooctane and one and two ring compounds.

The thermal diffusion behavior of linear alkanes is well described by a simple lattice model $(\mathrm{SLM})^{8}$. At the same time the SLM is not capable to describe the thermal diffusion behavior of branched alkanes because their thermodynamic parameters such as density, 
heat capacity and thermal expansion coefficient are not sensitive to the degree of branching. Recently, we investigated the influence of the degree of branching by RNEMD simulation ${ }^{12}$. The simulated Soret and mutual diffusion coefficients reproduce the experimental trend. However, the simulated values of $S_{\mathrm{T}}$ values are systematically $\approx 3 \times 10^{-3} \mathrm{~K}^{-1}$ lower than in

the experiment. The observed decrease of the magnitude of $S_{\mathrm{T}}$ for equimolar alkane/benzene mixtures with branching of the alkane can not be explained by mass and size effects. The effect of the molecular shape, which affects the liquid structure, as well as kinetic properties of the mixture, needs to be considered additionally. We have, however, not found a simple relation to take branching or, more generally, molecular shape, into account.

In this paper we extend our work by considering hydrocarbon/aromatic compound mixtures. Benzene was replaced by cyclohexane and two xylene isomers ( $p$-xylene and $o$-xylene). As a solute we used heptane, two of its isomers 2,3-dimethylpentane (2,3-DMP) and 2,4dimethylpentane (2,4-DMP). Experiments were performed using the thermal diffusion forced Rayleigh scattering technique. The experimental results were compared with RNEMD simulations. Additionally, we investigated the effect of intramolecular flexibility using RNEMD by introducing two double bonds for 2,3-DMP and 2,4-DMP e.g. considering two alkadienes: 2-methyl-3-methylenepent-1-ene (2,3-DMPEN) and 2,4-dimethylpenta-1,3-diene (2,4DMPEN), respectively.

\section{EXPERIMENTAL DETAILS}

\section{A. Sample preparation}

Heptane, o-xylene (98\%) and p-xylene (98\%) were purchased from Fluka; 2,3dimethylpentane (99\%) and 2,4-dimethylpentane (99\%) were ordered from Aldrich; cyclohexane (99.9\%) we got from LiChrosolv. Fig. 1 shows the chemical structure of the investigated molecules. For all mixtures the alkane mole fraction was adjusted by weighing the components. The TDFRS experiments require a small amount of dye in the sample. In this work, all samples contained approximately $0.002 \mathrm{wt} \%$ of the dye Quinizarin (Aldrich). This amount ensures a sufficient optical modulation of the grating but is small enough to avoid convection and contributions of the dye to the concentration signal. Before each TDFRS experiment, approximately $2 \mathrm{ml}$ of the freshly prepared solution were filtered through 0.2 


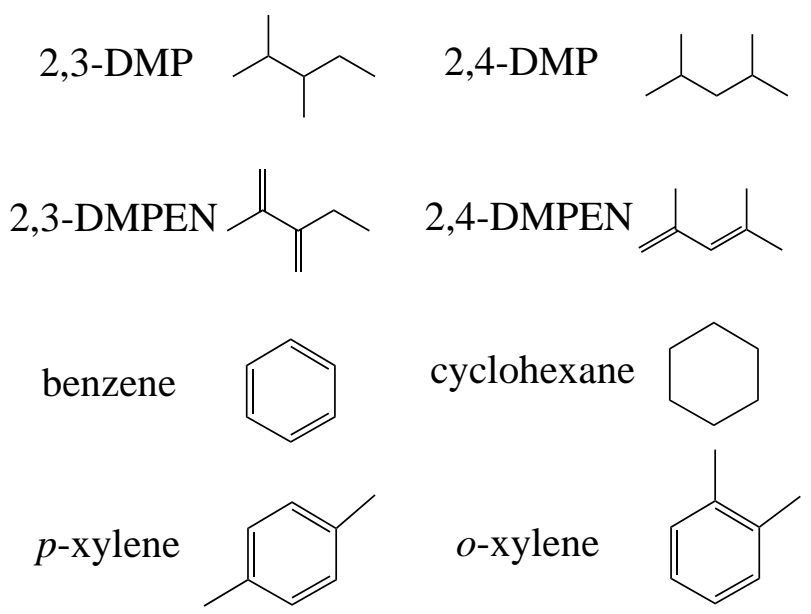

FIG. 1: Chemical structure of the investigated molecules. The branched alkanes (or alkadienes) are: 2,3-DMP (2,3-dimethylpentane), 2,4-DMP (2,4-dimethylpentane), 2,3-DMPEN (2-methyl-3methylenepent-1-ene) and 2,4-DMPEN (2,4-dimethylpenta-1,3-diene).

$\mu \mathrm{m}$ filter (hydrophobic PTFE) into an optical quartz cell with $0.2 \mathrm{~mm}$ optical path length (Helma) which was carefully cleaned from dust particles before usage.

\section{B. Refractive index increment measurements}

In order to determine the changes of the refractive index $n$ with composition $x$ at constant pressure $P$ and temperature $T,(\partial n / \partial x)_{P, T}$, for each hydrocarbon/aromatic ring compound mixture we measured the refractive index at different concentration around the equimolar mixture with an Anton Paar RXA 156 refractometer. The slope $(\partial n / \partial x)_{P, T}$ was then determined by linear interpolation. The temperature derivatives at constant pressure and composition, $(\partial n / \partial T)_{P, x}$, were determined from measurements with a Michelson interferometer ${ }^{13}$ in a temperature range of $3{ }^{\circ} \mathrm{C}$ above and below the temperature of the TDFRS experiment.

\section{TDFRS experiment and data analysis}

In our thermal diffusion forced Rayleigh scattering (TDFRS) experiments, the beam of an $\operatorname{argon-ion~laser}\left(\lambda_{w}=488 \mathrm{~nm}\right)$ is split into two writing beams of equal intensity which interfere 
in the sample cell (see Ref. [14] for a detailed description of the method). A small amount of dye is present in the sample and converts the intensity grating into a temperature grating, which in turn causes a concentration grating by the effect of thermal diffusion. Both gratings contribute to a combined refractive index grating, which is read out by Bragg diffraction of a third laser beam $\left(\lambda_{r}=633 \mathrm{~nm}\right)$.

The intensity $\zeta_{\text {het }}(t)$ of the signal depends on the transport coefficients and the index of refraction increments and may be expressed as

$$
\zeta_{\text {het }}(t)=1+\frac{(\partial n / \partial x)_{P, T}}{(\partial n / \partial T)_{P, x}} S_{\mathrm{T}} x(1-x)\left(1-e^{-q^{2} D t}\right) .
$$

where $q$ is the grating vector, whose absolute value is determined by the angle $\theta$ between two writing beams and the wavelength $\lambda_{w}$ :

$$
q=\frac{4 \pi}{\lambda_{w}} \sin \frac{\theta}{2}
$$

For the determination of the transport coefficients, Eq. (2) is fitted to the measured heterodyne signal using the independently measured contrast factors $(\partial n / \partial x)_{p, T}$ and $(\partial n / \partial T)_{p, w}$.

\section{COMPUTATIONAL DETAILS}

Reverse nonequilibrium molecular dynamics (RNEMD) method has been applied to investigate the thermal diffusion of binary mixtures of alkane (alkadiene) in $p$-xylene, $o$-xylene and cyclohexane. Lorentz-Berthelot mixing rules were employed for unlike nonbonded interactions. The force field parameters for the benzene ring of xylene were taken from Milano and Müller-Plathe ${ }^{15}$. The $\mathrm{C}-\mathrm{H}$ bonds were slightly polarized in order to reproduce the quadrupole moment. For the carbon and hydrogen atoms of the $\mathrm{CH}_{3}$ xylene group we used the following Lennard-Jones parameters: $\epsilon=0.22 / 0.15 \mathrm{~kJ} / \mathrm{mol}$ and $\sigma=0.3 / 0.245 \mathrm{~nm}$ respectively.

For alkanes, alkedienes and cyclohexane we used the TraPPE-UA ${ }^{16,17}$ force field. All $\mathrm{CH}_{n}$ groups were treated as individual atoms without taking into account electrostatic interactions. We would briefly like to mention the differences between the force fields for alkanes and alkedienes. For the $\mathrm{CH}_{2}$ and $\mathrm{CH}_{3}$ groups present in both alkane and alkediene we used the same Lennard-Jones parameters. The difference in shape due to the double bond we 
TABLE I: The comparison of the physical properties of $p$-xylene and $o$-xylene obtained from the simulation and experimental work.

\begin{tabular}{|l|c|c|c|c|}
\hline \hline \multirow{2}{*}{ property \system } & \multicolumn{2}{|c|}{$p$-xylene } & \multicolumn{2}{|c|}{ o-xylene } \\
\cline { 2 - 5 } & experiment & simulation & experiment & simulation \\
\hline density / $\mathrm{gmL}^{-1}$ & $0.857^{20}$ & $0.858 \pm 0.002$ & $0.876^{20}$ & $0.864 \pm 0.003$ \\
$\mathrm{H}_{\text {vap }} / \mathrm{kJmol}^{-1}$ & $42.38^{21}$ & $41.7 \pm 0.1$ & $43.43^{21}$ & $37.5 \pm 0.1$ \\
self diffusion $/ 10^{-5} \mathrm{~cm}^{2} \mathrm{~s}^{-1}$ & $4.1^{22}$ & $2.24 \pm 0.2$ & - & $1.78 \pm 0.2$ \\
\hline \hline
\end{tabular}

took into account by appropriate new LJ parameters of $\mathrm{sp}^{2}$ geometry ${ }^{17}$, angle and bond length changes, which lead to the expected different specific volume for alkediene compared to alkane. Only constrained bonds were used in the simulation, which were kept rigid by the SHAKE algorithm ${ }^{18,19}$. For the electrostatic interactions, the reaction field method has been used with a dielectric constant of 2.06 which is the average value of the alkanes and the xylenes. We found a good agreement between simulated and experimental values for the density, heat of vaporization and self diffusion of the xylene molecules (c.f. Table I). Unfortunately, we could not find any reliable experimental values for the self diffusion coefficient of $o$-xylene but it is in the same order of magnitude as for benzene molecule ${ }^{15}$.

All simulations were performed using the program YASP $^{23,24}$ with periodic boundary conditions in all directions. The time step was 2 fs. The nonbonded cutoff was $1.2 \mathrm{~nm}$ for the neighbour list and $1.1 \mathrm{~nm}$ for the interactions. The neighbour list was updated every 15 time steps. The temperature and pressure were in equilibrium molecular dynamic simulations kept at $298 \mathrm{~K}$ and $101.3 \mathrm{kPa}$ using the Berendsen method with coupling times of $0.2 \mathrm{ps}$ for the temperature and 5 ps for the pressure. For every system, the equilibration was performed for at least $4 \mathrm{~ns}$. After this period, the fluctuations of the total energy and density over 500 ps were in all cases below $2 \%$. Then, the cell was replicated three times in the direction of temperature gradient (in our case is $\mathrm{z}$ direction, means $\mathrm{L}_{x}=\mathrm{L}_{y}=\mathrm{L}_{z} / 3 \approx 4$ $4.2 \mathrm{~nm})$.

All RNEMD simulations were performed at constant NVT conditions with 960 molecules in the simulation box. The periodic system was divided into 20 slabs. The average temperature was kept at $298 \mathrm{~K}$. The temperature gradient was created by exchanging every $N=100$ steps the center of mass velocity vector of two molecules ("coldest" molecule in the 


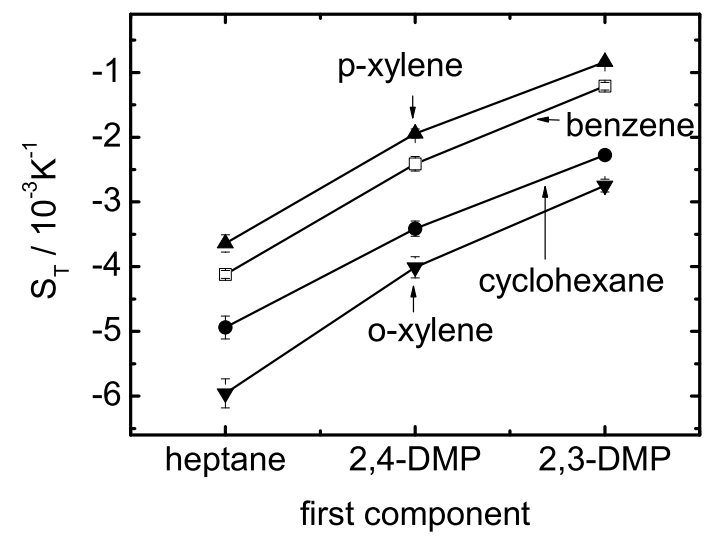

FIG. 2: The experimentally measured Soret coefficients for equimolar mixtures of some alkanes and alkadienes in different aromatic compounds at $T=30^{\circ} \mathrm{C}$. The data for hydrocarbon/benzene mixtures were taken from Polyakov et al. ${ }^{12}$.

hot slab one and the "hottest" molecule in the cold slab eleven) of the same type. The temperature profile was sampled every 101th step. After the concentration gradient is induced the Soret coefficient can be calculated (c.f. Eq. 1). For each simulation run two values of the Soret coefficient were calculated: from the nine slabs of the downward branch and from the nine slabs in the upward branch. The hottest and coldest slabs have been excluded from the analysis. The final value of $S_{\mathrm{T}}$ represents the average value, the error bars reflect the difference between $S_{\mathrm{T}}$ from downward and upward branches.

\section{RESULTS AND DISCUSSION}

\section{A. Experiment}

Fig. 2 shows the experimentally determined Soret coefficient for different hydrocarbon/aromatic compound mixtures. For all considered solvents the magnitude of $\mathrm{S}_{T}$ becomes smaller with increasing degree of branching of the first component and is also sensitive to the nature of the second component. This is the same trend which we observed for other heptane isomers in benzene ${ }^{12}$. It is remarkable, that all curves have the same shape and no intersection points were observed.

The obtained results can be analyzed within a phenomenological conception proposed by 
TABLE II: Physical properties for the solvents used in the analysis by Eq. 8: heat of vaporization at the boiling point ${ }^{25,26}$, density at room temperature ${ }^{25}$ and the principal moment of inertia ${ }^{27}$.

\begin{tabular}{|c|c|c|c|c|c|}
\hline solvent & $\begin{array}{l}\Delta H_{\text {vap }} / \\
\mathrm{kJ} / \mathrm{mol}\end{array}$ & $\begin{array}{c}\rho / \\
\mathrm{g} \cdot \mathrm{cm}^{-3}\end{array}$ & $\begin{array}{c}\mathrm{Ixx} \\
\AA^{2}\end{array}$ & $\begin{array}{l}\text { Iyy } \\
\AA^{2}\end{array}$ & $\begin{array}{l}\text { Izz } \\
\AA^{2}\end{array}$ \\
\hline heptane & 36.57 & 0.684 & 44 & 661 & 681 \\
\hline 2,4-DMP & 33.1 & 0.673 & 123 & 349 & 425 \\
\hline 2,3-DMP & 34.1 & 0.695 & 149 & 269 & 292 \\
\hline benzene & 33.83 & 0.874 & 82 & 821 & 164 \\
\hline cyclohexane & 33 & 0.774 & 117 & 117 & 203 \\
\hline$p$-xylene & 42.38 & 0.857 & 89 & 328 & 410 \\
\hline o-xylene & 43.43 & 0.876 & 149 & 220 & 362 \\
\hline
\end{tabular}

the group of Köhler ${ }^{28}$. They assumed that the Soret coefficient for a given mixture (of the component $\mathrm{A}$ in the component $\mathrm{C}$ ) is only determined by the difference in the properties of the pure mixing partners $\sigma^{A}$ and $\sigma^{C}$, respectively.

$$
S_{\mathrm{T}}^{A C}=\sigma^{A}-\sigma^{C}
$$

$\sigma$ might be interpreted as heat affinity. Thus, the Soret coefficient of the components A in the component $\mathrm{C}\left(\mathrm{S}_{T}^{A C}\right)$ can be calculated using $\mathrm{S}_{T}^{A B}$ and $\mathrm{S}_{T}^{C B}$ :

$$
S_{\mathrm{T}}^{A C}=S_{\mathrm{T}}^{A B}-S_{\mathrm{T}}^{C B}
$$

Fig. 3 shows a satisfactory agreement between the experimental values of the Soret coefficient $S_{\mathrm{T}}$ and the calculated $S_{\mathrm{T}}^{\text {calc }}$ using Eq. 4 .

In order to determine the heat affinities an overestimated linear equation of the form

$$
\mathbf{M} \vec{\sigma}=\overrightarrow{\mathbf{S}}_{\mathbf{T}}
$$

has to be solved. $\vec{\sigma}$ and $\overrightarrow{\mathbf{S}}_{\mathbf{T}}$ are vectors consisting of the heat affinities and Soret coefficients of the different solvents and $\mathbf{M}$ is a matrix consisting of $1,-1$ and 0 combining the corresponding solvents with their Soret coefficients and heat affinities. In order to determine the heat affinities we have to rewrite the equation system.

$$
\vec{\sigma}=\left(\mathbf{M}^{\mathrm{T}} \mathbf{M}\right)^{-1} \mathbf{M}^{\mathrm{T}} \overrightarrow{\mathbf{S}}_{\mathbf{T}}
$$




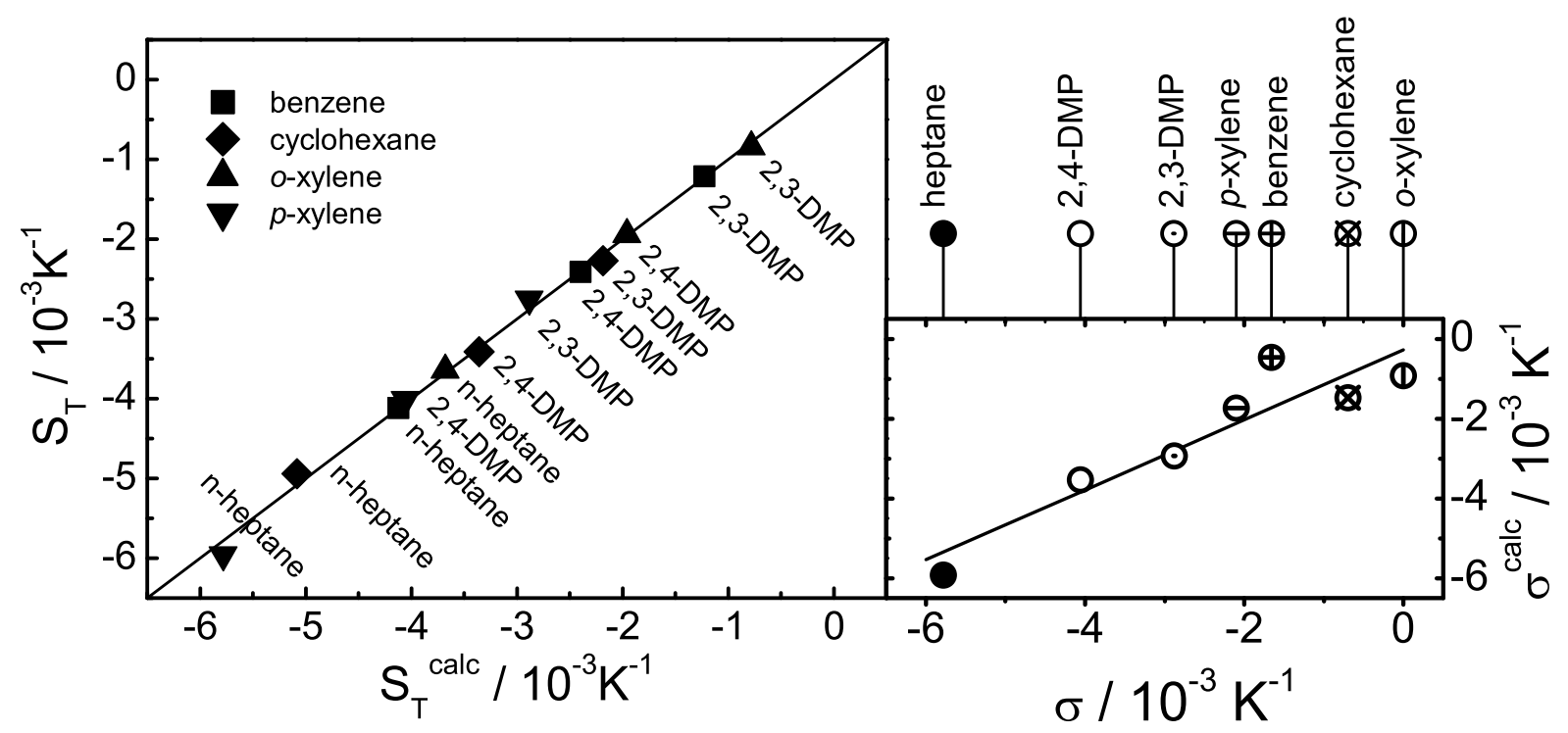

FIG. 3: Comparison of the experimental values of the Soret coefficient and the calculated ones using Eq. 5. The upper right part of the figure shows the heat affinity $\sigma$ of each solvent, which have been used to calculated $S_{\mathrm{T}}^{\text {calc }}$. In the lower right part of the figure $\sigma$ is correlated with the calculated heat affinity $\sigma^{\text {calc }}$ according to Eq.8 (black round symbols).

The heat affinities are only determined up to an arbitrary constant, which we have chosen to be zero for $o$-xylene, the substance with the lowest tendency to move to the warm side. The substance with the largest affinity to the cold is the asymmetric heptane. The calculated Soret coefficients are perfectly described by a straight line through the origin with a slope of $0.99 \pm 0.03$.

Our serie of heat affinities shows parallels with the logarithm of the separation factor $S$ given by Kramers and Broeders ${ }^{5}$. Also they found the lowest values for "log $S$ " for the two linear alkanes $n$-nonane and $n$-hexadecane followed by the branched iso-octane, one ring compounds (xylene, ethylcyclohexane, p-cymene) and two ring compounds (isopropylnapthalene, $\alpha$-methylnaphtalene).

The importance of the shape for the thermal diffusion in binary mixtures of two disk-like molecules was recently investigated for a mixture of benzene and cyclohexane by Debuschewitz and Köhler ${ }^{29}$. They correlated the Soret coefficient with the difference in mass and in 
the component of moment of inertia perpendicular to the molecular plane. In our case we have difficulties with the quantitative application of this conception because it is not clear which component of the moment of inertia needs to be considered for the alkane molecules. At the same time, it is clear that the observed "parallel shift" of the curves (c.f. Fig. 2) with the substitution of the solvent (benzene, cyclohexane, $p$ - or $o$-xylene) is related to the physical properties (difference in mass and moment of inertia) of the cyclic components. As a simple approach we related the heat affinity with the product of heat of evaporization $\Delta H_{\text {vap }}$ and the density $\rho$, the mass and the ratio of the largest to the smallest moment of inertia.

$$
\sigma=\gamma \cdot \Delta H_{v a p} \cdot \rho+\alpha \cdot m+\beta \cdot I^{\max } / I^{\min }
$$

The first term should account for the chemical contribution, the second term for the mass and the last term for the asymmetry of the molecule. The expression of the chemical contribution will certainly break down in the case of polar substances, but it might also loose its validity, if one considers other solvents. Also the contribution of the asymmetry of the substance becomes difficult in the case of larger and more flexible molecules.

The black round symbols in the lower right part in Fig. 3 show the correlation of $\sigma$ and $\sigma^{\text {calc }}$ according to Eq. 8 with $\gamma=1.74 \times 10^{-4} \mathrm{~mol} \mathrm{~cm} \mathrm{~cm}^{3} /(\mathrm{kJ} \mathrm{g} \mathrm{K}), \alpha=-6.57 \times 10^{-5} \mathrm{~mol} /(\mathrm{g} \mathrm{K})$ and $\beta=-2.38 \times 10^{-4} 1 / \mathrm{K}$. The straight line corresponds to a linear fit with a slope of 0.88 and an intercept of $2.74 \times 10^{-4}$. The correlation coefficient is only in the order of 0.93. Considering the component of moment of inertia perpendicular to the molecular plane $I_{z z}$ leads to slightly lower correlation coefficient of 0.89 . Especially the correlation for the organic ring compounds degrades, while the correlation of the alkanes slightly improves. Additionally, we can replace the chemical contribution by $\sqrt{\left(\Delta H_{v a p} \cdot \rho\right)}$, which corresponds to the Hildebrandt parameter $\delta$. This decreases the correlation coefficient further to 0.81 .

Finally, we would like to point out that we did not consider excess effects in Eq. 4 and 8 , which play an important role in the thermal diffusion behavior of liquid mixtures ${ }^{30}$. Due to the limited number of equimolar mixtures studied we were also not able to account for a change in composition. Whether this simple approach according to Eq. 8 holds also for other nonpolar systems needs to be investigated in the future for a large number of systems. 


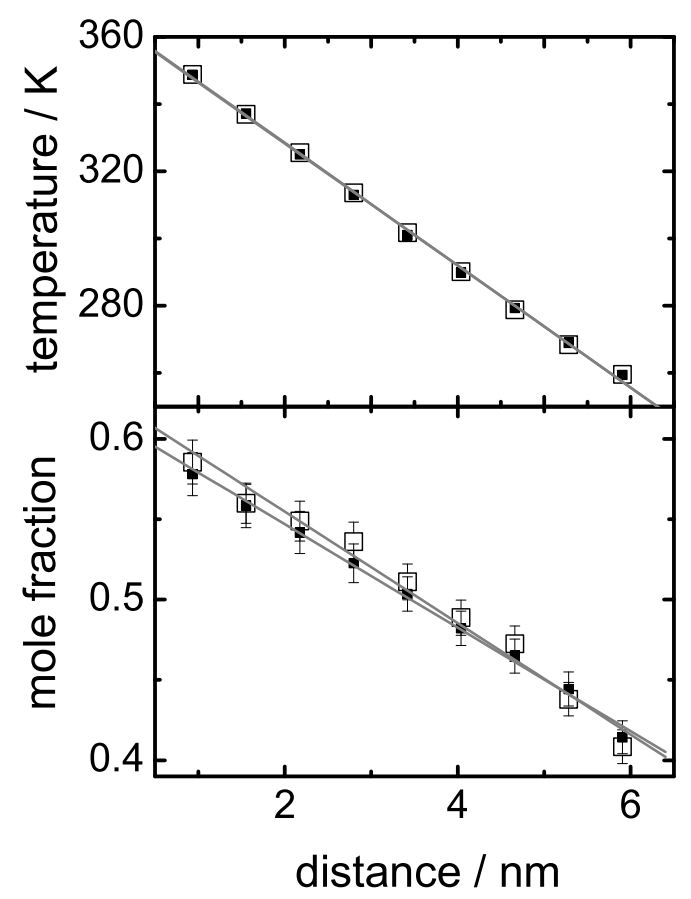

FIG. 4: The temperature and mole fraction profiles for $n$-heptane $/ p$-xylene mixture. The open and solid symbols refer to 9 slabs of the downward and upward branch in the simulation box.

\section{B. Simulation}

Additionally, we performed also simulations for the experimentally investigated mixtures. In order to study the influence of the rigidity of bonds we included also 2,3-DMPEN and 2,4-DMPEN. Fig. 4 shows a typical temperature and mole fraction profiles for equimolar mixtures of heptane in $p$-xylene. These profiles were found to be linear, so that the temperature and the concentration gradients can be easily calculated. The average fluctuation of the concentration is $8 \%$ and the temperature variation is $2 \mathrm{~K}$ in each slab.

Fig. 5 shows the simulated Soret coefficient for different alkane (alkadiene) /aromatic compound mixtures. For all considered solvents, the magnitude of the Soret coefficient becomes smaller with increasing the degree of branching of the first component. For the mixtures with xylenes the shapes of the curves are not sensitive to the nature of the second component and the substitution of $o$-xylene by $p$-xylene makes the magnitude of $\mathrm{S}_{T}$ smaller, what confirms the experimental trend (c.f. Fig. 2), but the simulation data are systematically to low. Or in other words their magnitude is too large, which means that 


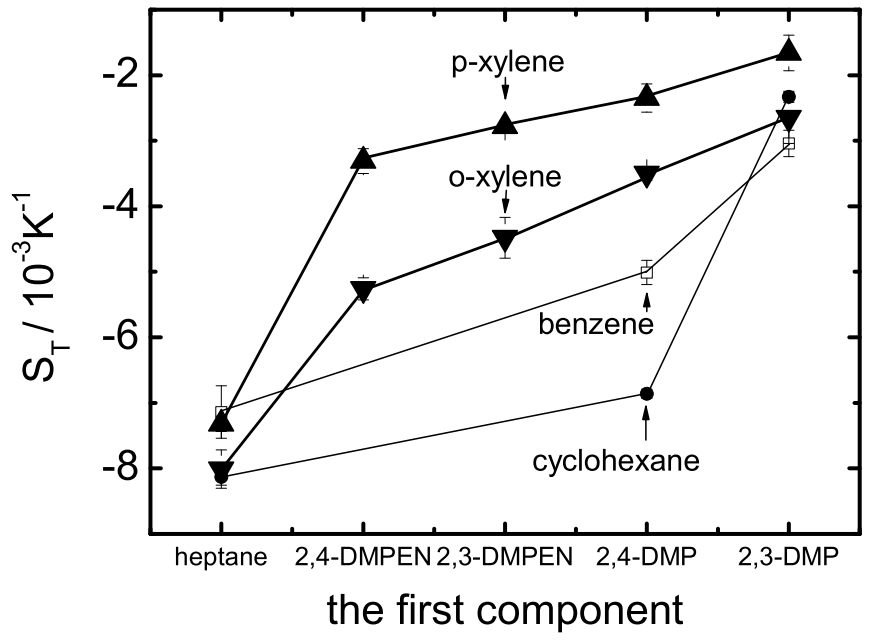

FIG. 5: The simulated Soret coefficients for equimolar mixtures of some alkanes and alkadienes in different solvents.

the simulations predict a larger separation of the compounds compared to the experiment. In same cases such as 2,3-DMP/heptane the values differ by a factor of 2 , but nevertheless the branching effect is also clearly visible in the simulations. If we look at the influence of the rigidity of bonds on the Soret coefficient by comparing the results for alkedienes and alkanes, we notice are stronger tendency for the alkedienes to move to the warm side. Fig. 5 shows some intersection points which were not observed in the experiment (c.f. Fig. 2). The possible reason could be an inconsistency of the used force fields. The Lennard Jones parameters used for benzene and cyclohexane were different from ones for $p$-xylene and $o$-xylene as explained in Sec. III.

In our previous work ${ }^{12}$ we have shown that the simple conception found for Lennard Jones (LJ) mixtures is not capable to explain the effect of branching for branched heptane/benzene mixtures. This approach can also not be used to explain the thermal diffusion behavior of alkane(alkadiene)/p-xylene and alkane(alkadiene)/o-xylene mixtures.

Although the validity of the Hildebrandt solubility parameter concept is not sufficient to describe the thermo diffusive motions ${ }^{31}$, there are several examples in the literature where a correlation has been found. For instance shows the Soret coefficient of spherical molecules ${ }^{4}$ a correlation with the energy density. And also for a thermosensitive polymer ${ }^{32}$ in different 


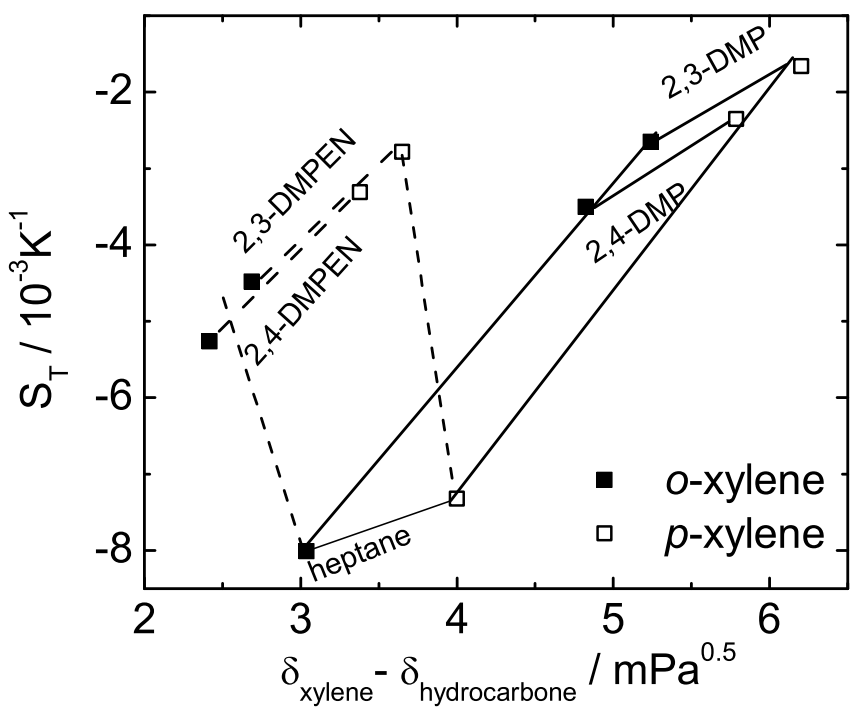

FIG. 6: The simulated Soret coefficients ( $p$-xylene: $\mathbf{\square}, o$-xylene: $\square$ ) plotted versus the difference in Hildebrandt parameter of the mixing partners.

alcohols the concentration at which the Soret coeffcient changes the sign is correlated with the Hildebrandt solubility parameter. Furthermore the simulations of Lennard-Jones liquids show a correlation between the Soret coefficient and the potential energy density of a species².

Fig. 6 shows the calculated Soret coefficient for ten xylene mixtures versus the difference in Hildebrandt parameters $(\triangle \delta)$ of the mixing partners. The Hildebrandt parameter has been calculated from equilibrium molecular dynamic simulations using the so-called nonbonded energy ${ }^{2,15}$. The magnitude of $S_{\mathrm{T}}$ becomes smaller with increasing $\triangle \delta$ for heptane, 2,3DMP and 2,4-DMP, what is not the case for the mixture of spherical LJ particles ${ }^{2}$. On the other hand if one compares 2,3-DMPEN and 2,4-DMPEN with heptane the opposite trend can be observed. One can expect, that a larger $\triangle \delta$ increases the difference between the mixing partners resulting in a larger value of the magnitude of $S_{\mathrm{T}}$. It is obvious that we can not confirm this simple correlation. Even for those nonpolar solvent the thermal diffusion behavior is guided by a delicate balance of cross and pure interactions between the mixing partners. 


\section{CONCLUSION}

The Soret coefficients of equimolar mixtures of heptanes ( heptane, 2,3-DMP and 2,4DMP) in cyclohexane, $p$-xylene and $o$-xylene were determined using TDFRS technique and RNEMD method. In the simulation we considered 2,3-DMPEN and 2,4-DMPEN, additionally. Both approaches show the decrease of the magnitude of $\mathrm{S}_{T}$ with increasing degree of branching, but the value determined by simulation is systematically to small, while its magnitude is too large. We could assign to each component a heat affinity, which allows the calculation of the Soret coefficient of the mixture and could be related to the heat of evaporisation, density, mass and the assymmetry of the pure component. The magnitude of the Soret coefficient does not increase with the difference in Hildebrandt parameters of the mixing partners as it is expected for the mixtures of Lennard Jones particles.

In order to gain a better understanding further investigations of the thermal diffusion on a microscopic level and a detailed molecular dynamic analysis of the orientation dynamic in equilibrium and non-equilibrium will be necessary. The goal will be to identify the important parameters and properties, which have the largest influence on the thermal diffusion behavior.

\section{ACKNOWLEDGMENTS}

We appreciate many fruitful discussions from Florian Müller Plathe an we thank Werner Köhler and Gerhard Wittko for stimulating discussions and sending us results prior publication. Malte Kleemeier we thank for reading the manuscript carefully. We thank also the Hochschulrechenzentrum der Technischen Universität Darmstadt for granting time on the IBM Regatta p575 machines (HHLR) located at the TU Darmstadt, Darmstadt, Germany and also to the John von Neumann-Institut for Computing (NIC) for granting time on IBM Regatta p690+ machines (JUMP) located at the ZAM Center, Jülich, Germany. Financial support due to the Deutsche Forschungsgemeinschaft grant Wi 1684 is gratefully acknowledged. 
* p.polyakov@fz-juelich.de

$\dagger$ e.rossinsky@theo.chemie.tu-darmstadt.de

$\ddagger$ s.wiegand@fz-juelich.de

1 S. Wiegand, J.Phys.:Condens. Matter 16, R357 (2004).

2 D. Reith and F. Müller-Plathe, J. Chem. Phys. 112, 2436 (2000).

3 G. Galliero, B. Duguay, J. P. Caltagirone, and F. Montel, Fluid Phase Equilibria 208, 171 (2003).

4 P. Polyakov, M. Zhang, F. Müller-Plathe, and S. Wiegand, J. Chem. Phys. 127, 014502 (2007).

5 H. Kramers and J. J. Broeder, Analytica Chimica Acta 2, 687 (1948).

6 A. L. Jones and E. C. Milberger, Industrial and Engineering Chemistry 45, 2689 (1953).

7 K. Shukla and A. Firoozabadi, Industrial Engineering Chemistry Research 37, 3331 (1998).

8 P. Polyakov, J. Luettmer-Strathmann, and S. Wiegand, J. Phys. Chem. B 110, 26215 (2006).

9 A. Leahy-Dios and A. Firoozabadi, J. Phys. Chem. B 111, 191 (2007).

10 P. Blanco, M. M. Bou-Ali, J. K. Platten, P. Urteaga, J. A. Madariaga, and C. Santamaria, J. Chem. Phys. 129, 174504 (2008).

11 A. Leahy-Dios, L. Zhuo, and A. Firoozabadi, J. Phys. Chem. B 112, 6442 (2008).

12 P. Polyakov, F. Müller-Plathe, and S. Wiegand, J. Phys. Chem. B 112, 14999 (2008).

13 A. Becker, W. Köhler, and B. Müller, Ber. Bunsen-Ges-Phys. Chem. 99, 600 (1995).

14 H. Ning, R. Kita, H. Kriegs, J. Luettmer-Strathmann, and S. Wiegand, J. Phys. Chem. B. 110, 10746 (2006).

15 G. Milano and F. Müller-Plathe, J. Phys. Chem. B 108, 7415 (2004).

16 G. Martin and J. Siepmann, J. Phys. Chem. B 102, 2569 (1998).

17 C. D. Wick, M. G. Martin, and J. I. Siepmann, J. Phys. Chem. B 104, 8008 (2000).

18 J. Ryckaert, G. Ciccotti, and H. Berendsen, J. Comput. Phys 23, 327 (1977).

19 F. Müller-Plathe and D. Brown, Comput. Phys. Commun. 64, 7 (1991).

20 Y. Marcus, The properties of Solvents, vol. 4 (John Wiley \& Sons, Chichester, 1999).

21 J. Chickos, S. Hosseini, and D. Hesse, J. Thermochim. Acta 249, 41 (1995).

22 J. Nafisi-Movaghar, J. Birks, and K. Naqvi, Proc. Phys. Soc. 91, 449 (1967).

23 F. Müller-Plathe, Comput. Phys. Commun. 78, 77 (1993). 
24 K. Tarmyshov and F. Müller-Plathe, J. Chem. Inf. Model. 45, 1943 (2005).

25 R. Weast, ed., CRC Handbook of Chemistry and Physics, vol. 58th ed (CRC Press Inc., New York, 1977).

26 J. S. Chickos and W. E. Acree, J. Phys. Chem. Ref. Data 32, 519 (2003).

27 Chem3d, ver. 10 (2006).

28 G. Wittko, Ph.D. thesis, University Bayreuth, Germany (2007).

29 C. Debuschewitz and W. Köhler, Phys. Rev. Lett, 87, 055901 (2001).

30 K. I. Morozov, Phys. Rev. E 79 (2009).

31 P. A. Artola, B. Rousseau, and G. Galliero, Journal of the American Chemical Society 130, 10963 (2008).

32 R. Kita, P. Polyakov, and S. Wiegand, Macromolecules 38, 4554 (2007). 